\title{
Study on Indicator Diagram Modifying of Diesel Engine with MATLAB Xiaohua Ye ${ }^{1, a}$ Fuqiu Wang ${ }^{2, b}$ \\ ${ }^{1}$ Qingdao Ocean Shipping Mariners College, Qingdao Shandong 266071, CHINA \\ ${ }^{2}$ Qingdao Ocean Shipping Mariners College, Qingdao Shandong 266071, CHINA \\ aemail, yexhqmc@126.com; bemail,wangfq@coscoqmc.com
}

Keywords: MATLAB software; diesel engine; indicator diagram; main engine

\begin{abstract}
Indicator diagram is an important factor to study working status of diesel engine for engineers. To large extent, it will affect the safety operation of the vessel whether the results of calculation and analysis are accurate or not. According to the analysis on the frequency spectrum of a certain type marine diesel engine indicator diagram, the indicator diagram curve could be more accurate by means of the MATLAB software whose principle is the low pass filter eliminating passage effects and modifying the deviation of top dead center by polytropic index method. The results show that the corrective method to the indicator diagram measured by the MATLAB software is feasible and reasonable.
\end{abstract}

\section{Introduction}

To engineers, indicator diagram is an important factor to study the improvement of the diesel engine working process and the calculation of the indicated power. ${ }^{[1]}$ The data should be processed as a result of some errors, such as unexpected error, fault error, pressure zero point calibration error, passage effect, position error of the TDC and so on which could result in discontinuity of measured Indicator curve and differential coefficient, and even more influence to the calculation of combustion heat release rate. Based on the homogenization of the measured indicator data and the zero point calibration process, this paper focuses on the study of passage effect elimination and top dead center correction by the MATLAB software.

\section{Elimination of indicator diagram passage effect}

Diesel engine indicator diagram is usually measured by an indicator. There is a certain passage space between the pressure transducer of the indicator and the combustion chamber. The pressure in the passage will result in frequency oscillation while measuring so the influence of the passage effect should be eliminated as to the measured cylinder pressure data. ${ }^{[2]}$ The traditional mathematical smoothing method is a weighted sliding average, which probably could filter out useful information while processing cylinder pressure, so it is necessary to develop the effective passage effect filter. According to the analysis on the frequency spectrum of the indicator diagram, this issue develops a low pass filter to eliminate the passage effect of the indicator diagram by the MATLAB software.

Figure 1 is an indicator diagram of a marine diesel engine whose revolution speed is $1000 \mathrm{r} / \mathrm{min}$ and power is $220 \mathrm{~kW}$. The Figure shows that the indicator diagram curve is not smooth in the high-pressure part and a little amplitude fluctuation exists. Although the system error, random error and human error can be eliminated by homogenization and 
careful measurement, the pressure data measured still leads to not only amplitude fluctuation but also phase distortion so that the indicator diagram measured could not be a true response to changes in cylinder pressure. The Fourier transform is carried out to obtain the frequency spectrum characteristics of the indicator diagram. Figure 2 shows that the energy of the signal is mainly concentrated in the low frequency band but there is still large signal energy when the frequency is between $1500-2000 \mathrm{~Hz}$. It maybe the high frequency energy

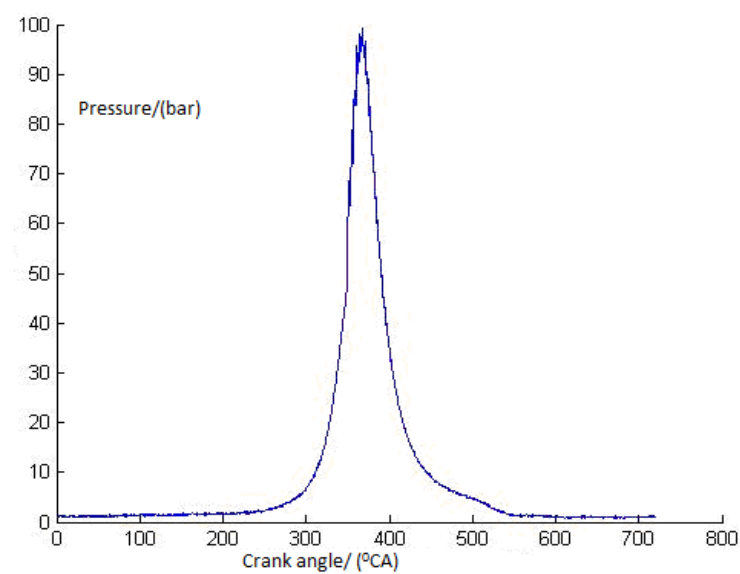

Fig.1 Indicator diagram before filtering

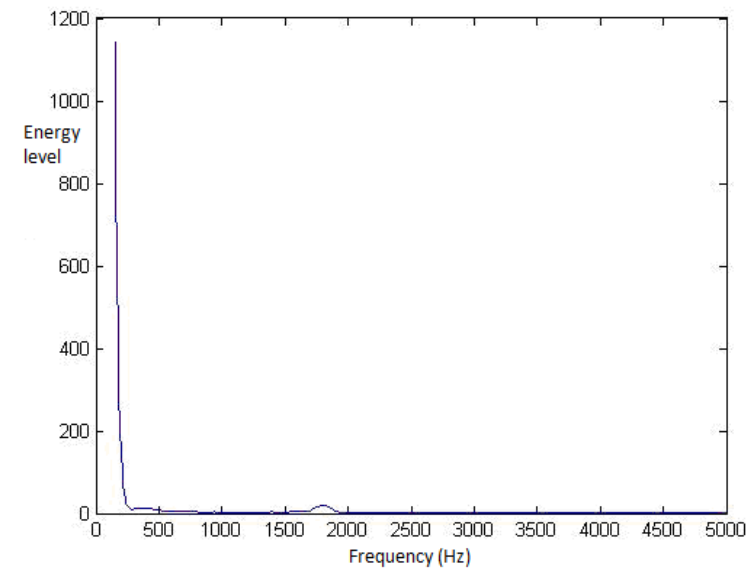

Fig.2 Frequency spectrum characteristics

generated by the passage effect.

\section{Calculation of inherent frequency of pressure measuring passage}

The formula for calculating the inherent frequency of the passage is:

$$
f=\frac{20 \cdot \sqrt{T}}{2 \cdot \pi} \cdot \sqrt{\frac{S}{L \cdot V x}}
$$

in the formula:

$\mathrm{f}$ - - Inherent frequency of the pressure measuring passage, $\mathrm{Hz}$;

$\mathrm{S}$ - Sectional area of the pressure measuring passage, $\mathrm{m}^{2}$;

$\mathrm{L}$ - L Length of the pressure measuring passage, $\mathrm{m}$;

$\mathrm{V}_{\mathrm{x}}$ - Volume of the cavity below the sensor, $\mathrm{m}^{3}$ 。

According to the formula (1), the inherent frequency of the pressure measuring passage is related to the geometry of the channel and the working condition of the diesel engine. As to the given testing passage, its structure no longer changes. However, the change of the working condition of diesel engine may lead to changes of temperature and pressure of working fluid in cylinder; Consequently, the inherent frequency could change in a reasonable range. While under the rated working condition of the diesel engine, the inherent frequency of passage effect is $1,300 \mathrm{~Hz}$ by formula (1) according to the transducer installation, passage size and estimated temperature within cylinder. The results of calculation are similar owing to the sound speed within the cylinder is hard to be 
determined according to the analysis to the spectrum of the indicator diagram, it could be indicated that the inherent frequency of the channel effect is above $1300 \mathrm{~Hz}$.

\section{Design of digital filter}

There are two working methods of the digital filter -- Frequency Domain Method and Time Domain Method. By means of the Frequency domain method, we discrete the signals by Fourier transform, and then analyze its spectral characteristic and filter the other frequency of the signals according to the desired frequency characteristics, and finally return the time domain signal by Fourier transform. The frequency domain selection characteristics and flexibility of this method is much better so we could filter the indicator diagram by the frequency domain method in this paper.

A filter is a method that enables a particular frequency component to pass, suppresses or attenuates other frequency components. The relationship among the output signal $y(n)$, the input $\mathrm{x}(\mathrm{n})$ and the impulse response $\mathrm{h}(\mathrm{n})$ is:

$$
y(n)=x(n) \cdot h(n)
$$

If the Fourier transform of $\mathrm{x}(\mathrm{n}), \mathrm{y}(\mathrm{n})$ exists, its frequency domain characteristic is:

$$
Y\left(e^{j w}\right)=X\left(e^{j w}\right) \cdot H\left(e^{j w}\right)
$$

In the formula:

$Y\left(e^{\mathrm{jw}}\right)$ - the frequency spectrum characteristics of the output sequence of the digital filter;

$X\left(e^{\mathrm{jw}}\right)$ - the frequency spectrum characteristics of the input sequence of the digital filter;

$H\left(e^{\mathrm{jw}}\right)$ - frequency domain response of digital filter。

In accordance with the formula (3), the spectrum of the input sequence $\mathrm{X}\left(e^{\mathrm{jw}}\right)$ is filtered to $X\left(e^{\mathrm{jw}}\right) H\left(e^{\mathrm{jw}}\right)$. According to the characteristics to the spectrum of input signal and the purpose of the signal process, the frequency domain response $H\left(e^{\mathrm{jw}}\right)$ can be selected so that the design performance could be achieved after filtering.

According to the functions of the filter, the filters can be divided into low-pass filter (LPF), high-pass filter (HPF), band-pass filter (BPF) and band-stop filter (BSF). In accordance with the above analysis, the filter of the diesel engine indicator diagram should be low-pass filter (LPF) and pass band cut-off frequency
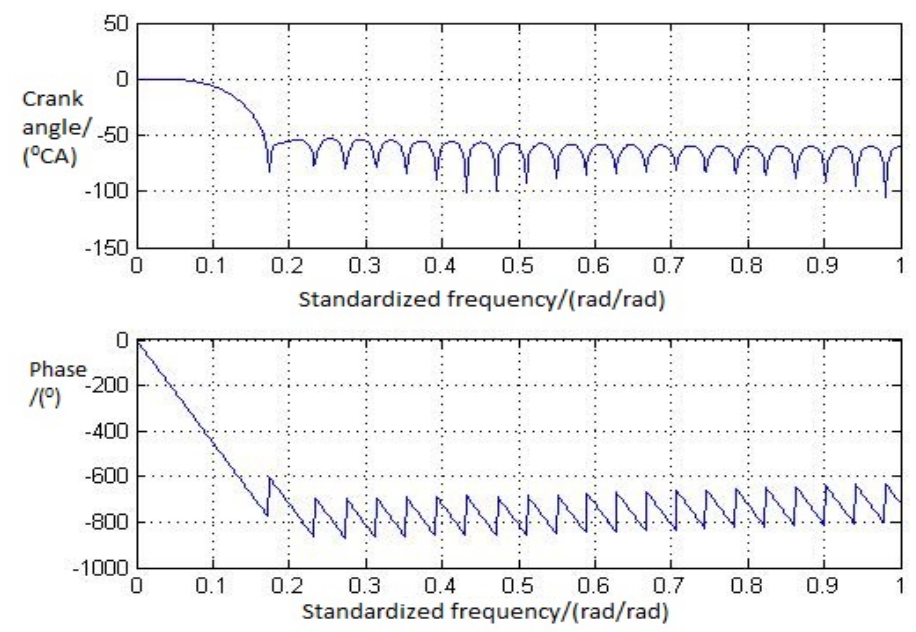

Fig.3 The amplitude frequency and phase frequency diagram of the designed FIR low-pass filter $1500 \mathrm{~Hz}$. In order to avoid the phase distortion after filtering, we can take the finite pulse 
corresponding digital filter. The applicable filters could be designed easily by the MATLAB toolbox. Figure 3 is about the amplitude frequency and phase frequency diagram of the designed FIR low-pass filter.

As shown in Figure 4, the indicator diagram of a certain type of diesel engine could be filtered by the above-mentioned digital filter in the rated working condition. Many burrs with small amplitude have been eliminated in the shown indicator diagram. By spectrum analysis to the filtered indicator diagram, the Frequency domain graph could be obtained as shown in Figure 5 and the high

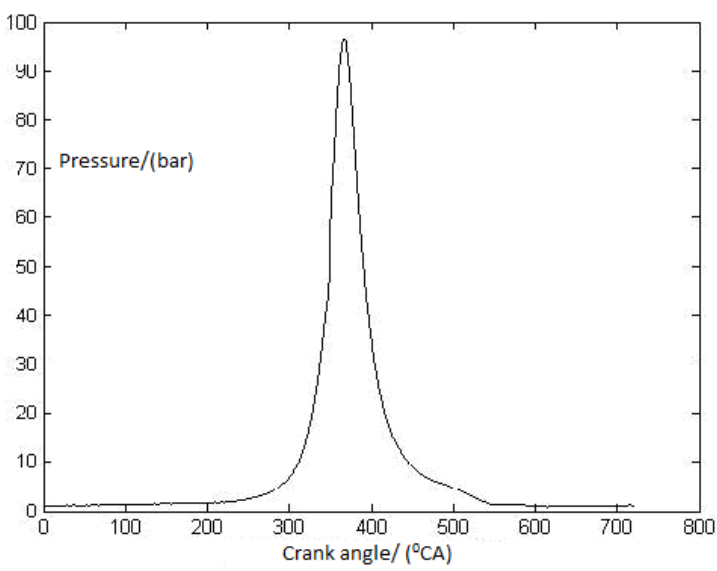

Fig.4 Indicator diagram after filtering

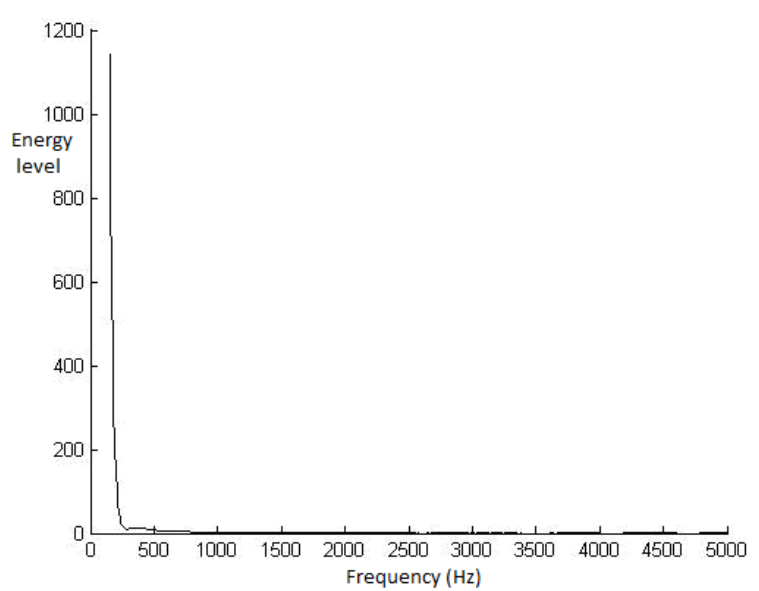

Fig.5 Frequency domain graph after filtering

frequency energy fluctuation above $1500 \mathrm{~Hz}$ is eliminated.

\section{Top dead center (TDC) correction of the indicator diagram}

While testing the indicator diagram of a diesel engine, the error of the TDC position will lead to the error of the instantaneous volume and volume change in the cylinder and then affect the heat release rate. The maximum deviation of the top dead center is $1^{\circ} \mathrm{CA}$ and correspondingly the maximum error of the heat release rate is about $\pm 5 \%$, so the deviation of the top dead center is the main factor which affects the calculation of heat release rate. The TDC position error is inevitable among various factors of process of indicator diagram measurement, so the position of the TDC should be modified. ${ }^{[3-5]}$

The correction of the top dead center is mainly carried out under pure compression conditions. The main methods are thermal equilibrium method, temperature commercial method, $p-\varphi$ curve symmetry discrimination method and polytropic index method. Thermal equilibrium method and temperature commercial method are both based on the energy analysis. The position error itself of the TDC in the indicator diagram affects the accuracy of the energy and could result in errors. The discrimination method on $\mathrm{p}-\varphi$ curve symmetry is not accurate as a result of leakage. Thus, the top dead center could be corrected by the polytropic index method in this paper.

In the ideal state, it is considered that the pure compression process and the expansion process of 
the diesel engine are adiabatic processes. According to the nature of the variable process, the polytropic index near the top dead center is continuous, then $\mathrm{dVc}=0, \mathrm{dp}_{\mathrm{c}}=0$. If there is an error in the position of the TDC, the calculated polytropic index is not continuous. Consequently, the position of the TDC could be corrected by the polytropic index method.

As to the reversible polytropic process, the basic state parameters of gas comply with the $P_{c} V_{c}^{n^{\prime}}=$ constant. The differential form of the above equation is $\frac{d p_{c}}{p_{c}}+n^{\prime} \cdot \frac{d V_{c}}{V_{c}}=0$. We can get the polytropic index formula:

$$
n^{\prime}=-\frac{d p_{c} / p_{c}}{d V_{c} / V_{c}}
$$

The indicator diagram in Fig. 6 is measured while cutting off oil to the single cylinder at the idle speed. It could be indicated that curves of the compression process and the expansion process are symmetrical.

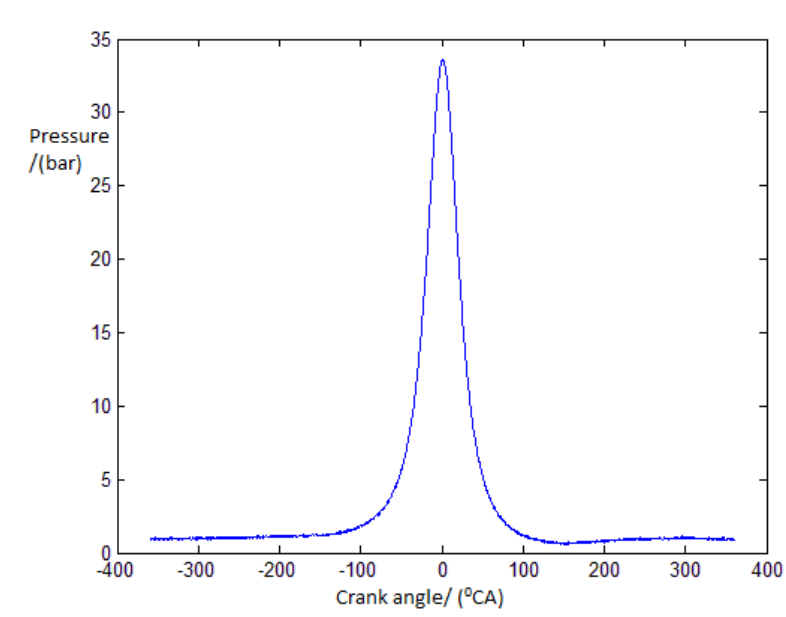

Fig.6 Indicator diagram

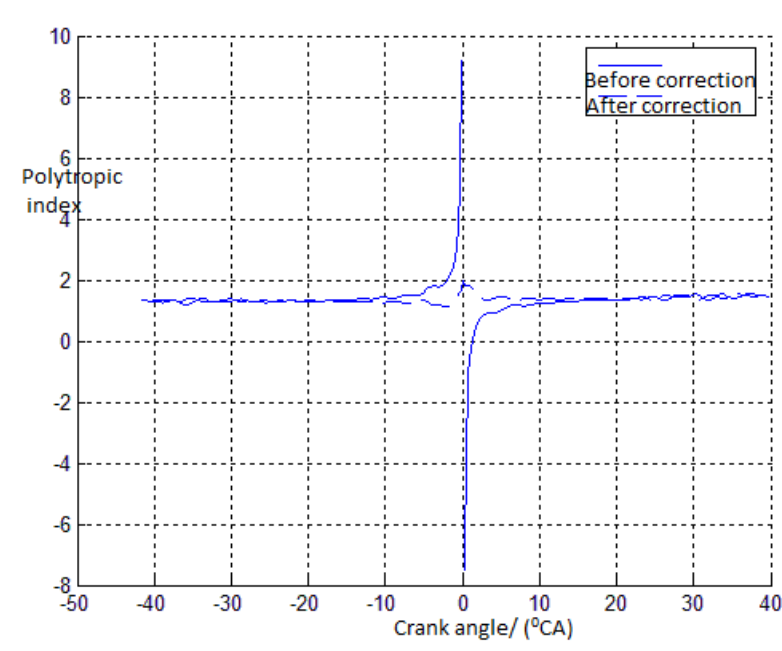

Fig.7 Polytropic index before and after correction

The polytropic index curve measured in the driving process is very sensitive to the position of the TDC. The criteria whether the correction of the TDC is better or not is: If the position of the TDC is set accurately, the change of the variable index near the TDC is small; If not, the mutation of the curve could exist. In figure 7, it could be shown that influence of the polytropic index to the deviation of the TDC before and after the correction of the indicator diagram while cutting off oil to the single cylinder at $1000 \mathrm{r} / \mathrm{m}$.

The deviation of TDC is related to heat transfer loss and air leakage loss. The offset of TDC is different because heat loss angle is different resulting from different heat transfer and air leakage loss in the cylinder under different revolution speed. So the TDC should be corrected by the pressure curve obtained at different speed. Fig. 8 is an indicator diagram measured from one diesel engine, through which the offset of TDC in different revolution speed could be obtained by the TDC correction with the polytropic index method. 


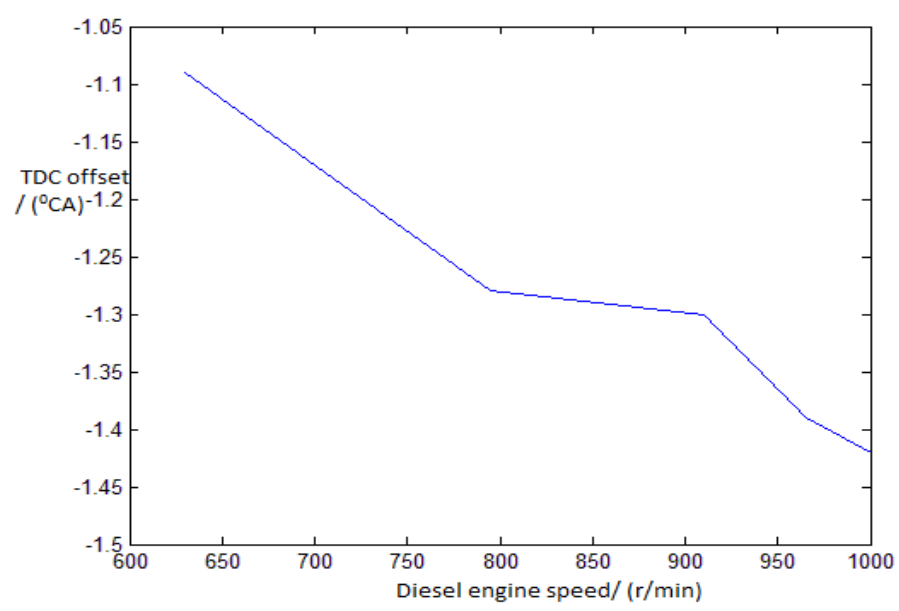

Fig.8 Offset of TDC in different revolution speed

\section{Conclusions}

With the MATLAB software, the more accurate indicator diagram curve could be obtained by TDC correction with the polytropic index method and elimination to the passage effect by the designed filter based on the homogenization and the pressure zero calibration treatment. The engineers could evaluate working condition of the diesel engine timely and accurately by the indicator diagram resulting from analysis by means of the data processing method; Moreover, they could also regulate the operating parameters as required.

\section{References}

[1]CAO Guo-hao, YU Yong-hua, YANG Jian-guo. Development of a Indicator Digram Measurement system Based on Microcontroller for a Marine Diesel Engine [J].Ship \& Ocean Engineering, 2010,39(1): 84-87.

[2]XU Lu-jie, NIE Zhi-bin. Eliminating Method of Indicator Diagram Channeling Effect for a Certain Type of Diesel Engine [J]. Ship \& Ocean Engineering, 2013,42(2): 74-76.

[3]CHANG Han-bao, DING Jia-song.A Study of Thermodynamic Method for TDC Correcting in the Indicator Diagram of Diesel Engines [J].Chinese Internal Combustion Engine engineering, 2003,24(4): 78-81.

[4]YU Yong-hua,YANG Jian-guo. Development of Performance Monitoring Analyzer for Marine Diesel Engine [J].Ship Engineering,2009,31(1) : 8-10.

[5]YIN Zi-bin ,LI Zhu-qing. Power Measurement with Indicator Diagram of on-board Diesel engine[J].Journal of Dalian Marine University,2005,31(3):5-8. 